\title{
Systematic versus sentinel-lymph-node-driven axillary-lymph-node dissection in clinically node-negative patients with operable breast cancer. Results of the GF-GS01 randomized trial
}

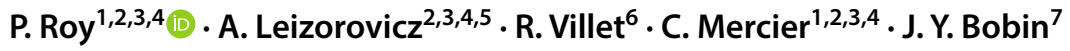

Received: 20 February 2018 / Accepted: 23 February 2018 / Published online: 10 March 2018

(c) The Author(s) 2018

\begin{abstract}
Purpose Sentinel-lymph-node (SLN) resection seems to minimize systematic axillary-lymph-node dissection (sALND) side effects in operated breast cancer patients. We explored whether SLN resection achieves similar therapeutic outcomes as sALND but with fewer side effects.

Methods A randomized, controlled, open-label trial with parallel-group design compared sALND restricted to cases with positive SLN biopsy (test arm, $n=774$ ) versus SLN biopsy followed by sALND (control arm, $n=770$ ).

Results The five-year overall survivals in control and test arms were 96.42 and $95.64 \%(P=0.2925)$. The estimated difference was nearly zero (precisely, $-0.79 \%$, one-tailed $95 \%$ confidence interval (CI) limit $-2.44 \%$ ). In a multivariate Cox model, the adjusted hazard ratio in the test arm was HR 0.81 (upper 95\% CI limit 1.17). Advanced age (HR 1.05 per additional year, CI [1.03-1.08]), negative progesterone receptor (HR 2.17 [1.35-3.45]), SLN metastasis (HR 1.69 [1.03-2.79]), and only one SLN identification technique (HR 4.14 [1.21-14.18]) were associated with lower survival. Patients with $\geq 1$ severe side effect at 1 month in control and test arms were $173 / 703=24.6 \%[21.5-28.0 \%]$ and $91 / 693=13.1 \%[10.7-15.9 \%](P<0.001)$. The estimated sensitivity of SLN biopsy (control arm) was 145/178 $=81.5 \%$ [74.8-86.7\%].

Conclusions Restricting ALND to cases with positive SLN biopsy does not affect the overall survival but reduces by $11.5 \%$ [7.5-15.6\%] $(P<0.001)$ the risk of severe short-time side effects of sALND.
\end{abstract}

Keywords Breast cancer $\cdot$ Clinical trial $\cdot$ Sentinel-lymph-node biopsy $\cdot$ Surgery $\cdot$ Survival

$\begin{array}{ll}\text { Abbreviations } \\ \text { ALND } & \text { Axillary-lymph-node dissection } \\ \text { BMI } & \text { Body Mass Index } \\ \text { CI } & \text { Confidence interval }\end{array}$

P. Roy

pascal.roy@chu-lyon.fr

1 Service de Biostatistique-Bioinformatique, Hospices Civils de Lyon, 162 Avenue Lacassagne, 69003 Lyon, France

2 Université de Lyon, Lyon, France

3 Université Lyon 1, Villeurbanne, France

4 Laboratoire de Biométrie et Biologie Évolutive, CNRS, UMR 5558, Villeurbanne, France

5 Service de pharmacologie clinique et essais thérapeutiques, Hospices Civils de Lyon, Bron, France

6 Service de chirurgie viscérale et gynécologique, Groupe hospitalier Diaconesses-Croix-Saint-Simon, Paris, France

7 Service de Chirurgie Oncologique, Hospices Civils de Lyon, Pierre-Bénite, France

\author{
GF-GS01 Acronym of the trial \\ HE Hematoxylin and eosin stains \\ HIC Immunohistochemical \\ HPS Hematin, phloxine, and saffron stains \\ HR Hazard ratio \\ OR Odds ratio \\ SBR Scarff-Bloom-Richardson \\ SLN Sentinel lymph node (n) or sentinel-lymph- \\ node (comp. n. or adj.)
}

\section{Introduction}

Systematic axillary-lymph-node dissection (ALND) is a procedure aimed to establish nodal status and guide adjuvant treatment indication to maximize survival and regional control of cancer in breast cancer patients. However, this procedure has been shown associated with short-term and long-term side effects in a substantial number of patients [1-3]. Sentinel-lymph-node (SLN) resection was proposed 
to minimize ALND side effects [4]. Within this context, the GF-GS01 trial was designed to establish whether SLN resection achieves similar therapeutic outcomes as ALND but with fewer side effects. The initial aim of the GF-GS01 trial was to demonstrate the non-inferiority of the test arm (ALND restricted to positive SLN) in terms of relapse-free survival. The trial expected fewer post-surgical complications.

\section{Methods}

\section{Trial design}

GF-GS01 is a randomized, controlled, open-label trial with a parallel-group design (ClinicalTrials.gov Identifier: NCT00144898). SLN resection was performed in both arms, whereas ALND was systematic in the control arm but restricted to SLN-positive patients in the test arm.

\section{Participants}

Patients were included in 70 centers in France between August 2003 and June 2007. To be eligible, women aged 18 or older had to present invasive breast cancer $\leq 30 \mathrm{~mm}$ at clinical examination or mammography, confirmed by needle (cytology) or micro/macro biopsy (histology), without clinical node involvement (N0) or organ metastasis (M0). An informed written consent was obtained from each participant.

\section{Axillary sentinel-lymph-node identification}

At least $25 \mathrm{~min}$ before entrance to the operating room, the patient was given an injection of one $\mathrm{mCi}$ of Technetium- $99 \mathrm{~m}$ colloidal rhenium sulfide $(1 \mathrm{~mL}$ followed by injection of $0.2 \mathrm{~mL}$ of air) intradermally for superficial tumors or in the gland close to the tumor for deep tumors. Injecting into the tumor was strictly forbidden. The skin was marked when radioactive lymph nodes were localized using a gamma probe, the arm being positioned in surgical posture. Patent blue (Gerbet, France) was injected in the operating room in the presence of an anesthetist, either a single intradermal injection of two $\mathrm{mL}$ in front of the tumor or two peritumoral injections of one $\mathrm{mL}$ each. Lymph nodes that were radioactive and/or blue were labeled as sentinel lymph nodes. An extemporaneous examination of the sentinel lymph node was performed only in case of suspicion of nodal involvement by the surgeon or the pathologist. Optionally, the internal mammary chain sentinel-lymph node could also be explored in the case of internal or medial tumors. Lymphoscintigraphy was optional and not subject to evaluation in this trial. Sentinel-lymph nodes were identified before being sent to the pathologist.

SLNs without detectable metastasis on paraffin stains [Hematoxylin and Eosin stains (HE), Hematin, Phloxine, and Saffron stains (HPS)] including serial node sectioning were analyzed using immunohistochemical (IHC) techniques. Non-SLNs were analyzed with HPS, completed with IHC techniques when a doubt persisted after HPS examination.

\section{Number of subjects needed (initial calculation)}

Under the alternative hypothesis of identical disease-free survival probabilities, 382 events (relapse or death) had to be observed to reject the null hypothesis of a hazard ratio of 1.35 in $90 \%$ of the studies ( $\beta=10 \%$ ), a type-one error rate $\alpha=5 \%$ (one-tailed) being retained. On the basis of a planned accrual period of 2 years, a 5-year follow-up of the last patient included, and an expected 5-year disease-free survival of $85 \%$ in the control arm, it was decided to randomize 2152 patients (1076 per arm). Under the hypothesis of detection of sentinel lymph node in $95 \%$ of the patients and a discovery of a metastatic SLN on extemporaneous examination in 5\% of cases, 2400 patients had to be included in the trial.

\section{Randomization}

When SLN was not macroscopically suspect on biopsy, the patients were randomly assigned to "test" and "control" arms in a 1:1 ratio (centralized computer randomization). Randomization was stratified on age at study entry $(\leq 50,>50$ years) and study center. Because masking was not possible due to the nature of the procedures, the coinvestigators had no information about the randomization process (mixture of blocks of various sizes).

\section{Follow-up}

Patients were planned to be monitored for overall survival, disease-free survival, and regional cancer control up to 5 years. An administrative request was made to obtain the official vital status of women at end of follow-up.

\section{Outcomes}

The initial primary endpoint of the trial was relapse-free survival. Secondary endpoints included sentinel false-negative rate (control arm), post-surgical complications during the first month and later, and overall survival. The first month post-surgical complications included axillary infection, axillary lymphedema, axillary hematoma, axillary bleeding, axillary paresthesia or intercostobrachial nerve injury, 
pain with arm movement, brachial plexus injury, and "other" complications.

The trial had to overcome several difficulties, i.e., lack of financial resources, decrease of investigators motivation, whereas there was no evidence of non-inferiority of the test arm in the literature. The follow-up of patients was not completed in several centers. Then, neither the primary endpoint nor the late side effects were available for the majority of the patients. At the blind review, the steering committee decided to change the primary endpoint into overall survival and restrict the analysis of the secondary endpoints to those available at 1 month of follow-up (i.e., the analysis of relapse-free survival was dropped). It was also decided to exclude seven centers ( 83 patients) because of unavailable follow-up data.

\section{Sample size}

The number of patients of the GF-GS01 non-inferiority trial was initially calculated for the disease-free survival endpoint. According to the steering committee, there was no justification for a new power calculation on the new primary endpoint after the closure of the trial.

\section{Statistical analysis}

No interim endpoint analyses were planned. The analyses were carried out according to the intent-to-treat principle.

Overall survival was analyzed as primary outcome. The follow-up of still-alive patients was censored at the date of the vital status request (June 18, 2012). Data on patients lost to follow-up were censored at the date of last follow-up. The survival curves were estimated using Kaplan-Meier methods [5] and compared using the log-rank test. The estimated difference in the probability of 5-year survival was calculated together with the corresponding lower limit of the onetailed $95 \%$ confidence interval (CI). A frailty proportional hazard regression model $[6,7]$ was fitted, with "center" as random effect. Survivals of patients randomized in the test and the control arms were compared using Wald test and the estimated hazard ratio (HR) (test arm versus control arm) with its $95 \%$ CI upper limit.

A secondary analysis of survival was performed including, in the model, variables "treatment arm" and "age", and testing the following variables: pathological tumor size, Scarff-Bloom-Richardson (SBR) grade, SLN status, Body Mass Index (BMI), tumor location, histological type, hormone receptors, metastatic embolization, SLN identification procedure, and non-surgical treatments (radiotherapy, adjuvant chemotherapy, adjuvant hormone therapy). The assumption of proportional hazard was checked by analyzing Schoenfeld residuals. A $P$ value $<5 \%$ in Wald test was considered for statistical significance.
Post-surgical complications at 1 month were described by arm and compared using Fisher exact tests. The occurrence of at least one complication was compared between test and control arms by fitting a mixed effects unconditional logistic regression model, with a fixed effect put on variable "arm", random effects put on variable "center", and "arm-center" interaction. This model included variable "age" and tested variables "BMI" and "history of shoulder disease".

The proportions of patients with positive SLN were estimated in each arm (with the corresponding 95\% CI) and compared using a Fisher exact test.

In the control arm, the factors associated with axillarylymph-node involvement and SLN involvement were analyzed fitting unconditional logistic regression models that included systematically "age" (as fixed effect) and "center" (as random effect). The analysis investigated the proportion of false negatives, i.e., the proportion of patients with negative SLN among those with positive ALND. Candidate risk factors for a false-negative result were analyzed. The type-1 error rate was fixed at $\alpha=0.05$ in all analyses.

The analyses were performed with SAS/STAT software, version 9.1.3 for Windows and the survival package of $R$ software, version 2.13.0 (http://www.r-project.org/).

\section{Results}

\section{Participants flow}

Figure 1 presents a description of the trial. The study randomized 1627 women. After exclusion of 83 patients from seven centers, 1544 patients were left for the statistical analysis: 770 in the control arm and 774 in the test arm. Protocol violations included non-compliance with eligibility criteria (five patients in the control arm vs. six in the test arm), disagreement between randomization and actual axilla treatment (one vs. four patients, respectively), and stratification error (13 vs. 12 patients, respectively).

\section{Baseline data}

Patient and tumor characteristics at inclusion are shown in Table 1. The distributions of the baseline characteristic were similar in the two arms. In all patients, history of shoulder disease was reported by $2.7 \%$ of the patients and the WHO performance status was excellent in $84.1 \%$. Technetium-99 m colloidal rhenium sulfide plus patent blue dye plus lymphoscintigraphy were used for axillary sentinellymph-node detection in $84.9 \%$ of all cases. In $81.6 \%$ of all cases, the tumor did not exceed $2 \mathrm{~cm}$ on pathology laboratory examination. Receptors for estrogen and progesterone were positive in 87 and $75 \%$ of all tumors, respectively. 


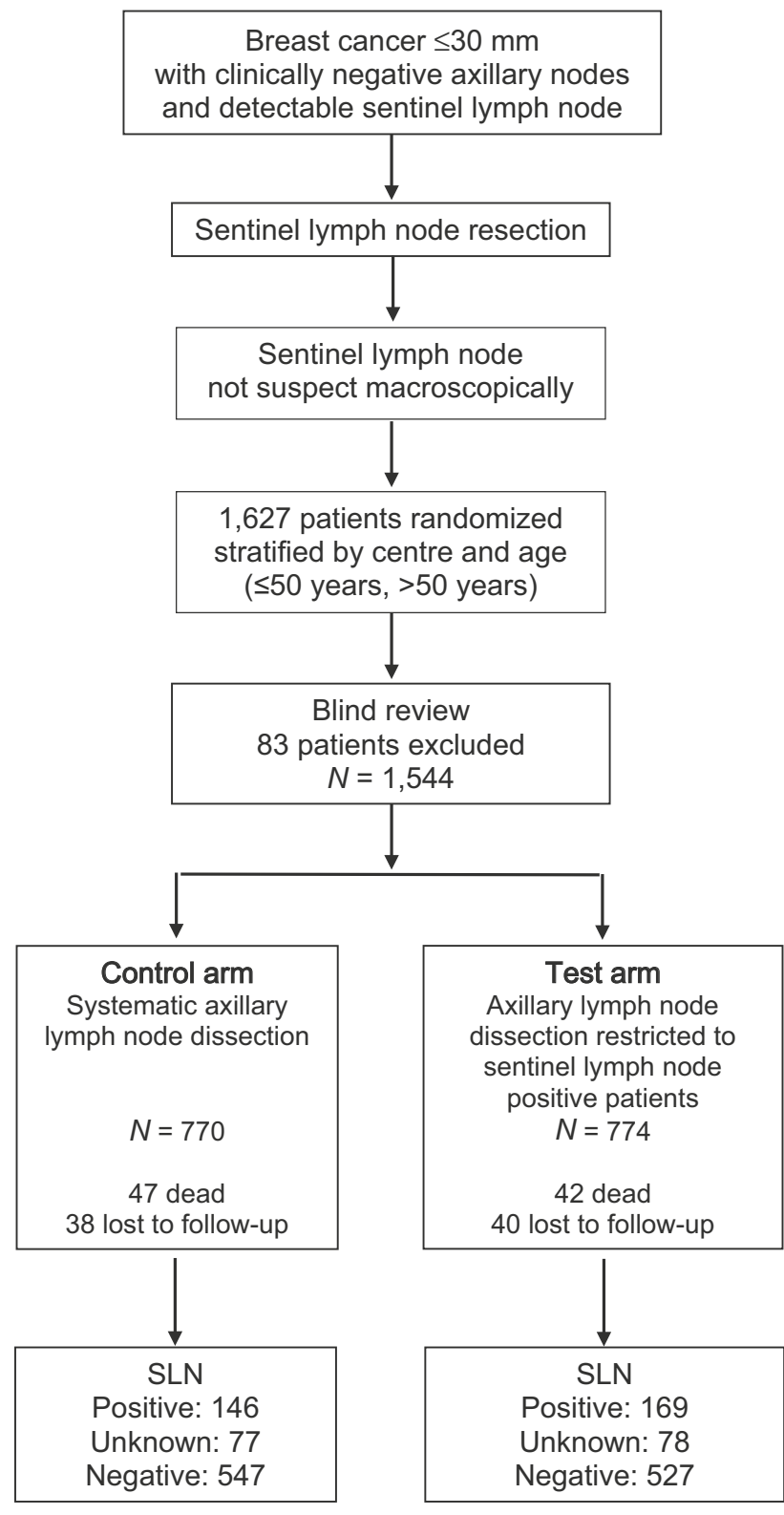

Fig. 1 Flow chart of GF-GS01 trial

\section{Main endpoint}

At the cut-off date (June 18, 2012), 89 patients were deceased and 78 lost to follow-up. The estimated overall survival probabilities were similar in the two study arms. Figure 2 shows that Kaplan-Meier overall survival curves superimpose. The estimated 5-year overall survival was $96.42 \%$ (95\% CI 95-98\%) in the control arm versus $95.64 \%$ (95\% CI 94-97\%) in the test arm. The null hypothesis of identical survivals between the two treatment arms could not be rejected (log-rank Chi-square test $=0.2983$, one-tailed $P$ value $=0.2925$ ). The estimated 5-year difference in survival probability (test arm minus control arm) was $-0.79 \%$, and the corresponding lower limit of the corresponding onetailed $95 \%$ CI was $-2.44 \%$. When a frailty proportional hazard regression model adjusted on age and center (random effect) was fitted, the hazard ratio for the test arm was HR 0.91 with an upper $95 \%$ CI limit (one-tailed) of 1.31 .

In the secondary analysis of survival, the independent prognostic factors associated with mortality were advanced age (HR 1.05 per additional year, 95\% CI 1.03-1.08), negative test for progesterone receptor (HR 2.17, 95\% CI 1.35-3.45), SLN metastasis (HR 1.69, 95\% CI 1.03-2.79), and the use of only one axillary sentinel-lymph-node identification technique (HR 4.14, 95\% CI 1.21-14.18). Factor Scarff-Bloom-Richardson grade III (HR 1.69, 95\% CI 0.99-2.87) was close to statistical significance. The adjusted hazard ratio for the test arm was 0.81 with an upper $95 \% \mathrm{CI}$ limit (one-tailed) of 1.17 (Table 2).

\section{Post-surgical complications at one month}

The prevalence of short-term complications was significantly lower in the test arm than in the control arm (Table 3). The estimated absolute differences in prevalence between the control and the test arm were $12.1 \%$ (95\% CI 8.1-16.1\%) for axilla seroma, $17.6 \%$ (95\% CI 13.2-22.0\%) for axillary paresthesia or intercostobrachial nerve injury, and $13.9 \%$ (95\% CI 9.6-18.2\%) for pain on arm movement. The proportion of patients presenting at least one severe side effect at 1 month was $173 / 703=24.6 \%(95 \%$ CI $21.5-28.0 \%)$ in the control arm versus and $91 / 693=13.1 \%(95 \% \mathrm{CI}$ $10.7-15.9 \%$ ) in the test arm; i.e., the estimated difference in prevalence was $11.5 \%$ (95\% CI 7.5-15.6\%, $P<0.001)$. The proportion of patients presenting at least one side effect at 1 month was $405 / 703=57.6 \%$ (95\% CI 54-61\%) in the control arm versus $224 / 693=32.3 \%$ (95\% CI 29-36\%) in the test $\operatorname{arm}(P<0.001)$. When a mixed effects unconditional logistic regression model was fitted, neither BMI nor history of shoulder disease was retained as independent risk factors of presenting at least one complication. Between-center heterogeneity led to introduce an arm-center interaction term. The prevalence of patients presenting at least one side effect at 1 month decreased with age (OR 0.99 per additional year, 95\% CI 0.98-1.00) and was importantly reduced in the tested arm (mean OR 0.30, 95\% CI 0.22-0.42, between-center $95 \%$ credible interval: 0.16-0.62).

\section{Proportions of patients with positive sentinel-lymph node}

The prevalence of positive SLN in all patients was $315 / 1389=22.7 \%(95 \%$ CI $20.5-25.0 \%) ; 146 / 693=21.1 \%$ in the control arm; and 169/696 $=24.3 \%$ in the test arm. The estimated difference (3.2\%) was not significant with $95 \% \mathrm{CI}$ -1.3 to $+7.8 \%(P=0.172)$. 
Table 1 Patient characteristics at baseline

\begin{tabular}{|c|c|c|c|}
\hline \multirow[t]{2}{*}{ Variable at inclusion and modality } & Systematic ALND & $\begin{array}{l}\text { ALND restricted to } \\
\text { positive SLN }\end{array}$ & Total \\
\hline & $N=770$ & $N=774$ & $N=1544$ \\
\hline \multicolumn{4}{|l|}{ Patient characteristics } \\
\hline Age & $59.2 \pm 10.9(696)$ & $59.6 \pm 11.2(693)$ & $59.4 \pm 11.0(1389)$ \\
\hline Body Mass Index & $24.9 \pm 4.5(670)$ & $24.6 \pm 4.3(673)$ & $24.8 \pm 4.4(1343)$ \\
\hline History of shoulder disease & $20 / 706(2.8 \%)$ & $18 / 700(2.6 \%)$ & $38 / 1406(2.7 \%)$ \\
\hline History of familial breast cancer & 76/704 (10.8) & $91 / 703(12.9)$ & $167 / 1407(11.9)$ \\
\hline History of familial ovary cancer & $11 / 703(1.6)$ & 6/703 (0.9) & $17 / 1406(1.2)$ \\
\hline Hormone replacement therapy & $84 / 699(12.0)$ & $89 / 695(12.8)$ & $173 / 1394(12.4)$ \\
\hline WHO performance status $/ n$ & $/ 663$ & $/ 663$ & $/ 1326$ \\
\hline 0 & $563(84.9)$ & $552(83.3)$ & $1115(84.1)$ \\
\hline 1 & $83(12.5)$ & $93(14.0)$ & $176(13.3)$ \\
\hline 2 & $11(1.7)$ & $9(1.4)$ & $20(1.5)$ \\
\hline 3 & $4(0.6)$ & $1(0.2)$ & $5(0.4)$ \\
\hline 4 & $2(0.3)$ & $8(1.2)$ & $10(0.8)$ \\
\hline \multicolumn{4}{|l|}{ Tumor clinical presentation } \\
\hline Breast side & 1706 & $/ 704$ & $/ 1410$ \\
\hline Right & $331(46.9)$ & $335(47.6)$ & $666(47.2)$ \\
\hline Left & $375(53.1)$ & $369(52.4)$ & $744(52.8)$ \\
\hline Breast clinical aspect $/ n$ & $/ 697$ & $/ 694$ & $/ 1391$ \\
\hline Normal & $550(78.9)$ & $539(77.7)$ & $1089(78.3)$ \\
\hline Adipose & $104(14.9)$ & $115(16.6)$ & $219(15.7)$ \\
\hline Fibrocystic mastopathy & $39(5.6)$ & $33(4.8)$ & $72(5.2)$ \\
\hline Other & $4(0.6)$ & $7(1.0)$ & $11(0.8)$ \\
\hline Tumor location $/ n$ & 1705 & 1705 & $/ 1410$ \\
\hline Upper-outer & $311(44.1)$ & $348(49.4)$ & $659(46.7)$ \\
\hline Lower-outer & $98(13.9)$ & $75(10.6)$ & $173(12.3)$ \\
\hline Upper-inner & 139 (19.7) & $118(16.7)$ & $257(18.2)$ \\
\hline Lower-inner & $48(6.8)$ & $63(8.9)$ & $111(7.9)$ \\
\hline Several/other & $109(15.5)$ & $101(14.3)$ & $210(14.9)$ \\
\hline Clinical tumor size $/ n$ & $/ 699$ & 1703 & $/ 1402$ \\
\hline T0 & $166(23.7)$ & $170(24.2)$ & $336(24.0)$ \\
\hline $\mathrm{T} 1$ & $439(62.8)$ & $454(64.6)$ & $893(63.7)$ \\
\hline $\mathrm{T} 2$ & $92(13.2)$ & $77(11.0)$ & $169(12.1)$ \\
\hline Other & $2(0.3)$ & $2(0.3)$ & $4(0.3)$ \\
\hline \multicolumn{4}{|l|}{ Tumor preoperative management } \\
\hline \multicolumn{4}{|l|}{ Mammography } \\
\hline Stellar lesion & $315 / 572(55.1)$ & $296 / 562(52.7)$ & $611 / 1134(53.9)$ \\
\hline Nodular lesion & 297/561 (52.9) & $291 / 560(52.0)$ & $588 / 1121(52.5)$ \\
\hline Microcalcifications & 94/498 (18.9) & 90/504 (17.9) & $184 / 1002(18.4)$ \\
\hline Architectural distortion & $79 / 493(16.0)$ & $112 / 511(21.9)$ & 191/1004 (19.0) \\
\hline Other & $21 / 443(4.7)$ & $20 / 445(4.5)$ & $41 / 888(4.6)$ \\
\hline Localization by harpoon & 220/692 (31.8) & $219 / 690(31.7)$ & $439 / 1382(31.8)$ \\
\hline Micro/macro biopsy & $567 / 692(81.9)$ & $534 / 685(78.0)$ & 1101/1377 (80.0) \\
\hline Cytology & 201/663 (30.3) & $202 / 652(31.0)$ & $403 / 1315(30.6)$ \\
\hline \multicolumn{4}{|l|}{ Primitive tumor management } \\
\hline Type of surgery & $/ 697$ & $/ 698$ & $/ 1395$ \\
\hline Radical mastectomy & $15(2.2)$ & $15(2.1)$ & $30(2.2)$ \\
\hline Lumpectomy & $682(97.8)$ & $683(97.9)$ & $1,365(97.8)$ \\
\hline Pathological staging & $/ 688$ & $/ 692$ & $/ 1380$ \\
\hline Microinvasive & $4(0.6)$ & $5(0.7)$ & $9(0.7)$ \\
\hline
\end{tabular}


Table 1 (continued)

\begin{tabular}{llll}
\hline Variable at inclusion and modality & Systematic ALND & $\begin{array}{l}\text { ALND restricted to } \\
\text { positive SLN }\end{array}$ & Total \\
& $N=770$ & $N=774$ & $N=1544$ \\
\hline pT1a & $44(6.4)$ & $42(6.1)$ & $86(6.2)$ \\
pT1b & $183(26.6)$ & $198(28.6)$ & $381(27.6)$ \\
pT1c & $327(47.5)$ & $323(46.7)$ & $650(47.1)$ \\
pT2 $<3 \mathrm{~cm}$ & $102(14.8)$ & $101(14.6)$ & $203(14.7)$ \\
pT2 $>3 \mathrm{~cm}$ & $13(1.9)$ & $14(2.0)$ & $27(2.0)$ \\
Others & $15(2.2)$ & $9(1.3)$ & $24(1.7)$ \\
SBR & $/ 663$ & $/ 671$ & $/ 1334$ \\
I & $196(29.6)$ & $223(33.2)$ & $419(31.4)$ \\
II & $345(52.0)$ & $326(48.6)$ & $671(50.3)$ \\
III & $122(18.4)$ & $122(18.2)$ & $244(18.3)$ \\
Estrogen receptors (IHC) & $/ 683$ & $/ 675$ & $/ 1358$ \\
Positive & $596(87.3)$ & $587(87.0)$ & $1183(87.1)$ \\
Negative & $87(12.7)$ & $88(13.0)$ & $175(12.9)$ \\
Progesterone receptors (IHC) & $/ 679$ & $/ 673$ & $/ 1352$ \\
Positive & $510(75.1)$ & $503(74.7)$ & $1013(74.9)$ \\
Negative & $169(24.9)$ & $170(25.3)$ & $339(25.1)$ \\
SLN technique & $/ 701$ & $/ 699$ & $/ 1400$ \\
Tec or PB & $9(1.3)$ & $13(1.9)$ & $22(1.6)$ \\
(Tec or PB) + LS & $45(6.4)$ & $46(6.5)$ & $91(6.5)$ \\
Tec + PB & $47(6.7)$ & $51(7.3)$ & $1189(84.9)$ \\
Tec + PB + LS & $600(85.6)$ & $589(84.3)$ & \\
\hline & & & $98.0)$ \\
\hline
\end{tabular}

Results are expressed as mean $\pm \mathrm{SD}(n)$, numerator/denominator $(n)$, or $n(\%)$

$A L N D$ axillary-lymph-node dissection, $S B R$ Scarff, Bloom, and Richardson grading system, $I H C$ immunohistochemistry, $S L N$ sentinel lymph node, $T e c$ technetium-99m, $P B$ patent blue, $L S$ lymphoscintigraphy

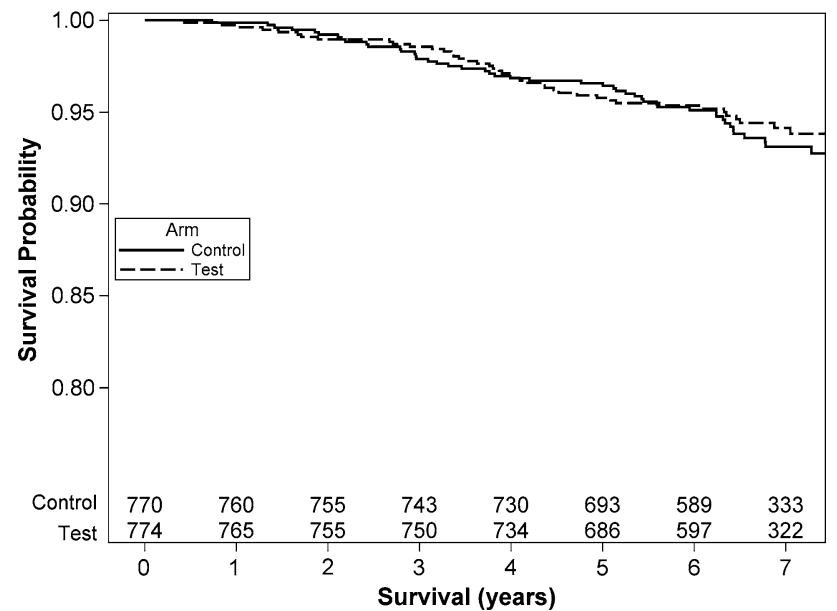

Fig. 2 Overall survival curves for test and control arms

\section{Probability of positive axillary lymph node and positive SLN (control arm)}

Positive axillary node (SLN or non-SLN) were observed in $180 / 680=26.5 \%$ (95\% CI $23.2-30.0 \%$ ) in the control arm.
One SLN-positive patient had a non-SLN unknown status and one non-SLN-positive patient had an SLN unknown status.

The probability of positive axillary lymph node increased together with tumor size (OR 1.93 per additional $\mathrm{cm}, 95 \% \mathrm{CI}$ 1.53-2.44) and negativity of tumor estrogen receptors (OR $2.18,95 \%$ CI 1.16-4.08) but decreased together with age at diagnosis (OR 0.98 per additional year, 95\% CI 0.96-0.99) and inner location of the tumors, particularly upper-inner tumor (OR 0.49, 95\% CI 0.29-0.83) (Table 4). Similar results were observed when factors associated with sentinellymph-node involvement were analyzed (Table 4).

\section{Probability of false-negative SLN (control arm)}

SLN and non-SLN were both positive in 41 patients and both negative in 499 patients, whereas 104 patients were SLN positive/non-SLN negative, and 33 patients were SLN negative/non-SLN positive. The estimated sensitivity of the SLN was $145 / 178=81.5 \%$ (95\% CI $74.8-86.7 \%$ ), and the corresponding probability of false-negative result was estimated at 33/178 $=18.5 \%$ (95\% CI 13.3-25.2\%). No significant risk factor was associated with the probability of a false 
Table 2 Results of the multivariate analysis. Hazard ratios for each explanatory variable modality

\begin{tabular}{lrlr}
\hline Variable and modality & \multicolumn{1}{l}{$N$} & HR $(95 \% \mathrm{CI})^{\mathrm{a}}$ & $P$ value $^{\mathrm{b}}$ \\
\hline Age (years) & 1364 & $1.05(1.03-1.08)$ & $<0.001$ \\
Age $^{2}$ & 1364 & $1.00(1.00-1.00)$ & 0.127 \\
SBR & & & 0.054 \\
$\quad$ I-II & 1124 & 1 & \\
III & 240 & $1.69(0.99-2.87)$ & 0.001 \\
Progesterone receptors & & & \\
Positive & 1024 & 1 & 0.039 \\
$\quad$ Negative & 340 & $2.17(1.35-3.45)$ & \\
SLN metastasis & & & 0.024 \\
$\quad$ Negative & 1053 & 1 & \\
Positive & 311 & $1.69(1.03-2.79)$ & \\
SLN identification & & & 0.173 \\
$\quad$ Tec + PB) \pm LS & 1346 & 1 & \\
Only Tec or PB & 18 & $4.14(1.21-14.18)$ & \\
Arm & & & \\
Control & 682 & 1 & \\
Test & 682 & $0.81(\mathrm{NA}-1.17)$ & \\
\hline
\end{tabular}

$H R$ hazard ratio, SBR Scarff, Bloom, and Richardson grade, Tec technetium-99m, $P B$ patent blue, $S L N$ sentinel lymph node, $L S$ lymphoscintigraphy

${ }^{a}$ One-tailed 95\% CI for clinical trial arm, two-tailed 95\% CI for other variables

${ }^{\mathrm{b}}$ Wald Test. One-tailed for clinical trial arm, two-tailed for other variables negative, whereas a non-significant trend was observed for larger tumors and lower-outer locations (Table 5).

\section{Discussion}

In operable breast cancer patients, the present phase III trial compared axillary-lymph-node dissection restricted to cases with sentinel lymph node (SLN) positive versus sentinel lymph node plus systematic axillary-lymph-node dissection (control arm) in terms of overall survival (primary endpoint) and post-surgical complications (secondary endpoints).

When the trial was initiated, the issue was important and few randomized clinical trials were designed to provide an answer. Overall survival was not considered to be different between the two arms; indeed, the hazard ratio for the test arm was 0.91 (unadjusted) with an upper 95\% CI limit of 1.31, and 0.81 (adjusted) with an upper 95\% CI limit of 1.17. Similar results were observed in two other randomized trials designed to answer the same question. In the Milanese trial [8], 516 patients were randomized according to the present trial design but the major endpoint was the occurrence of axillary metastasis. The Milanese trial reported 38 deaths and a 10-year overall survival of $93.5 \%$ (95\% CI 90.3-96.8\%) in the test arm versus $89.7 \%$ (95\% CI 85.5-93.8\%) in the control arm (log-rank test, $P=0.15)$. In the USA/Canadian NSABP B-32 trial [9], 309 deaths were reported among 3986 women with follow-up information; the estimated 5-year and 8-year overall survivals were 95.0 and $90.3 \%$, respectively, in the test arm versus 96.4 and $91.8 \%$ in the control arm, and the estimated unadjusted hazard ratio was 1.2 (95\% CI $0.96-1.50, P=0.12)$. The design of a third trial, Almanac (UK) [10], was close to the present one; 1031 patients were randomized into two arms
Table 3 Comparison of the prevalence of post-surgical complications at 1 month between test and control arm

\begin{tabular}{lccrr}
\hline First month side effects & Systematic ALND & $\begin{array}{l}\text { ALND restricted } \\
\text { to positive SLN } \\
N=693\end{array}$ & $\begin{array}{l}\text { Prevalence differ- } \\
\text { ence (95\% CI) }\end{array}$ & $P$ value $^{\mathrm{a}}$ \\
& $N=703$ & $8(1.2 \%)$ & 0.645 \\
\hline Axillary infection & $11(1.6 \%)$ & $73(10.5 \%)$ & $12.1 \%(8.1-16.1)$ & $<0.001$ \\
Axillary seroma & $159(22.6 \%)$ & $17(2.5 \%)$ & & 0.354 \\
Axillary hematoma & $12(1.7 \%)$ & $1(0.1 \%)$ & & 1.000 \\
Axillary bleeding & $1(0.1 \%)$ & $93(13.4 \%)$ & $17.6 \%(13.2-22.0)$ & $<0.001$ \\
Axillary paresthesia or intercosto- & $218(31 \%)$ & & & \\
$\quad$ brachial nerve injury & $192(27.3 \%)$ & $93(13.4 \%)$ & $13.9 \%(9.6-18.2)$ & $<0.001$ \\
Pain on arm movement & $0(0 \%)$ & $1(0.1 \%)$ & & 0.497 \\
Serratus anterior nerve injury & $38(5.4 \%)$ & $33(4.8 \%)$ & & 0.627 \\
Other complications & $173(24.6 \%)$ & $91(13.1 \%)$ & $11.5 \%(7.5-15.6)$ & $<0.001$ \\
Severe short-time side effects $(\geq 1)$ & $405(57.6 \%)$ & $224(32.3 \%)$ & $25.3 \%(20.1-30.5)$ & $<0.001$ \\
Short-time side effect $(\geq 1)$ & &
\end{tabular}

$A L N$ axillary lymph nodes (sentinel LN plus others), $A L N D$ axillary-lymph-node dissection, $S L N$ sentinel lymph node

${ }^{a}$ Two-tailed Fisher exact test 
Table 4 Multivariate analysis of factors associated with axillarylymph-node involvement and sentinel-lymph-node involvement (control arm)

\begin{tabular}{|c|c|c|c|c|c|c|}
\hline \multirow[t]{2}{*}{ Variable } & \multicolumn{3}{|c|}{ ALN involvement } & \multicolumn{3}{|c|}{ SLN involvement } \\
\hline & $N$ & OR $(95 \% \mathrm{CI})^{\mathrm{a}}$ & $P^{\mathrm{b}}$ & $N$ & OR $(95 \% \mathrm{CI})^{\mathrm{a}}$ & $P^{\mathrm{b}}$ \\
\hline Age (year) & 668 & $0.98(0.96-0.99)$ & 0.009 & 667 & $0.97(0.95-0.99)$ & 0.003 \\
\hline Tumor size $(\mathrm{cm})$ & 668 & $1.93(1.53-2.44)$ & $<0.001$ & 667 & $1.72(1.35-2.19)$ & $<0.001$ \\
\hline \multicolumn{7}{|l|}{ Tumor location } \\
\hline Upper-outer & 291 & 1 & & 291 & 1 & \\
\hline Lower-outer & 95 & $1.38(0.82-2.30)$ & 0.226 & 95 & $1.13(0.65-1.96)$ & 0.675 \\
\hline Upper-inner & 134 & $0.49(0.29-0.83)$ & 0.008 & 133 & $0.48(0.27-0.86)$ & 0.013 \\
\hline Lower-inner & 47 & $0.54(0.24-1.26)$ & 0.154 & 47 & $0.73(0.31-1.68)$ & 0.453 \\
\hline Several/other & 101 & $0.69(0.40-1.21)$ & 0.198 & 101 & $0.71(0.40-1.28)$ & 0.259 \\
\hline \multicolumn{7}{|l|}{ Estrogen receptors } \\
\hline Positive & 585 & 1 & & 584 & 1 & \\
\hline Negative & 83 & $2.18(1.16-4.08)$ & 0.016 & 83 & $2.11(1.08-4.15)$ & 0.030 \\
\hline
\end{tabular}

$A L N$ axillary lymph nodes (sentinel plus others), SLN sentinel lymph nodes

${ }^{\mathrm{a}}$ Two-tailed $95 \% \mathrm{CI}$

${ }^{\mathrm{b}}$ Wald test
Table 5 Factors associated with false-negative sentinel-lymph-node results

\begin{tabular}{llll}
\hline Variable & $N$ & OR $(95 \% \mathrm{CI})^{\mathrm{a}}$ & $P$ \\
\hline Age & 178 & $1.02(0.98-1.06)$ & 0.275 \\
Tumor size (cm) & 178 & $1.55^{\mathrm{b}}(0.93-2.59)$ & 0.099 \\
Tumor location & & & \\
Upper-outer & 87 & 1 & \\
Lower-outer & 34 & $2.14(0.84-5.47)$ & 0.114 \\
Upper-inner & 24 & $1.02(0.35-3.02)$ & 0.968 \\
Several/other & 33 & $0.94(0.29-3.08)$ & 0.921 \\
\hline
\end{tabular}

a Two-tailed 95\% CI

${ }^{\mathrm{b}} \mathrm{Per}$ additional $\mathrm{cm}$

(a test arm $=$ sentinel lymph node + axillary clearance or axillary radiotherapy in case of positive SLN and a control arm $=$ SLN + systematic axillary-lymph-node dissection) and the follow-up was restricted to 18 months because the major aim of the trial was assessing the patients' quality-oflife. In Almanac, at 12 months after surgery, seven deaths occurred in each arm.

Because of a less aggressive therapy in the test arm and the nature of the major endpoint criteria, a non-inferiority trial design was retained for the GF-GS01 trial, the Milanese trial, and the NSABP B-32 trial. One difficulty in such a trial design is providing a difference (or a ratio) to be rejected (which corresponds to rejection of the null hypothesis) or a threshold of equivalence for the difference in point estimate (or ratio). The Milanese trial was designed to reject a $5 \%$ difference or more in the proportion of axillary nodal metastases at 5 years. After a mean follow-up of 95 months, only 2 axillary metastases were observed, a much lower rate than expected, which did not allow a clear conclusion on the main endpoint of the trial [8]. The NSABP B-32 trial was designed to declare equivalence upon a $2 \%$ difference in survival or less between the two treatment arms among sentinel-node-negative patients [9]. Initially based on disease-free survival, the retained null hypothesis in the present GF-GS01 trial was a hazard ratio of 1.35 or more (See Number of subjects needed-initial calculation). In non-inferiority trials, the estimated upper limit of the $95 \%$ CI of the effect size is more informative than the level of significance. The estimated upper limits of the $95 \%$ CIs of the HRs of the GF-GS01 trial (1.17) and the NSABP B-32 trial (1.49) were almost close (note that the confidence intervals were onesided in GF-GS01 trial and two-sided in NSABP B-32 trial).

A positive SLN was observed in $169 / 696=24.3 \%$ of the patients in the test arm versus $146 / 693=21.1 \%$ of the patients in the control arm. This positivity rate of SLN in the control arm was smaller than the one observed in the NSABP B-32 trial $(694 / 2672=26.0 \%$ [11] or in the Milanese trial $(83 / 257=32.3 \%)$ [12]; however, here, IHC techniques were systemically used in case of negative sentinel lymph nodes, whereas they were used only for confirmation of suspected metastases in the NSABP B-32 or the Milanese trial. This result is not surprising because, here, the sensitivity of the SNL was $81.5 \%$ and its specificity was $100 \%$ by construction [13]. The corresponding estimated falsenegative rate was $33 / 178=18.5 \%(95 \%$ CI $13.3-25.2 \%)$, which is higher than the rates observed in the NSABP B-32 trial $(75 / 766=9.8 \%)[11]$, the Milanese trial $(8 / 91=8.8 \%)$ [12], or a meta-analysis (7.3\%) performed on 7754 patients of whom 3132 had nodal involvement [14]. Here, it is interesting to note that, in 14 out of 33 false-negative cases, only one sentinel lymph node was resected and that the mean number of sentinel lymph nodes resected in the control arm (i.e., 2.2) is comprised between the mean numbers found in 
the Milanese trial (1.7) [12] and in the NSABP B-32 trial (2.9) [11].

The proportion of short-time side effects is substantially different between the control and the tested arm (57.6\% vs. $32.3 \%$, respectively), the estimated absolute difference in prevalence being $25.3 \%$ (95\% CI $20.1-30.5 \%, P<0.001$ ). Differences in prevalence of axillary seroma (12.1\%), axillary paresthesia or intercostobrachial nerve injury (17.6\%), and pain on arm movement (13.9\%) contributed greatly to this difference. A comparison regarding the prevalence of side effects between the 100 first consecutive patients included in each arm of the Milanese trial showed that axillary pain, paresthesias on the operated side, and alteration of arm mobility at 6 and 24 months were more frequent in the control than in the test arm [12]. In the NSABP B-32 trial, the 12-month ipsilateral arm symptom mean score was 3.6 in the control arm versus 2.5 in the test $\operatorname{arm}(P=0.006)$ [15].

\section{Conclusions}

In the field of breast cancer without clinical node involvement or organ metastasis, the GF-GS01 trial is the second randomized trial in terms of number of women included in a comparison of axillary-lymph-node dissection restricted to SLN-positive patients with SLN biopsy followed by systematic axillary-lymph-node dissection. This GF-GS01 trial confirms that the former procedure reduces the risk of severe short-time side effects attributable to systematic axillary dissection by $11.5 \%$, without affecting the overall survival. Results from similar trials are welcome to provide a more accurate estimation of the effect size.

\section{Data availability}

The data that support the findings of this study are not publicly available. They are available on request after the agreement of the study scientific board.

Acknowledgements The authors are thankful to the following collaborators: Marmie S, MD; Tunon De Lara C, MD; Bussieres E, MD; Avril A, MD; Gioanni G, MD; Mage P, MD; Constantopoulos P, MD; Tribondeau P, MD; Le Touze O, MD; Mautalen F, MD; Delest A, MD; Larue-Charlus S, MD; Soffray F, MD (Bordeaux); Bernard JD, MD; Puyuelo L, MD; Cavalier B, MD; Manenc JL, MD; Farnarier J, MD; Berot JE, MD (Toulouse); Van Den Akker M, MD; Villet R, MD*; Salet-Lizee D, MD (Paris); Salvat J, MD (Thonon-les-Bains); Bobin JY, MD*; Charitanski H, MD; De Saint-Hilaire P, MD; Guimont I, MD; Awada A, MD (Lyon); Azuar P, MD (Grasse); Olivero JF, MD; Lanvin D, MD (Cagnes/mer); Bonnier P, MD; Brandone JM, MD; Conte M, MD; Houvenaeghel G, MD (Marseilles); Audrin O, MD; Beedassy B, MD (Toulon); Minguet C, MD (Sallanches); Chavrier F, MD (Annemasse); Miannay E, MD (Nantes); De Rochambeau B, MD; De Watteville JC, MD (Brou/Chantereine); Peltier JP,
MD (Castres); Rousseau E, MD (Dax); Joyeux P, MD (Bayonne); Flipo B, MD; Raoust I, MD; Legrand D, MD (Nice); Cohen M, MD (Aubagne); Miramand B, MD (Avignon); Routiot T, MD; Collet J, MD (Le Mans); Waeles JL MD (Mougins); Crozet B, MD (Sainte Colombe-Lès-Vienne); Seffert P, MD (Saint-Etienne); Toccanier JF, MD (Saint-Julien-en-Genevois); Blanchot J, MD (Rennes); Vaini-Elies V, MD (Aix-En-Provence); Gabelle P, MD; Bernard P, MD (Grenoble); Melchior J, MD (Saint-Avold); Garin B, MD (Sarcelles); and Routiot T, MD (Nancy). The authors also thank Evelyne Gauthier, Joëlle Gillet, and Michel Lièvre for their work at the coordinating center, Magalie Hervé for the data management, and Jean Iwaz (Hospices Civils de Lyon) for the revisions of the final drafts of the manuscript.

Funding The GF-GS01 trial was supported by the French Ministry of Health (Programme Hospitalier de Recherche Clinique, PHRC 2002).

\section{Compliance with ethical standards}

Conflict of interest The authors declare that they have no conflict of interest.

Ethical statements Trial GF-GS01 was carried out after approval from local institutional review boards and in accordance with assurances filed with and approved by the French Ministère de la Santé et des Affaires Sociales. Informed consent was obtained from all individual participants included in the study. The trial is registered under number (NCT00144898) in clinical.trial.gov and number RECF0322 in the database of the French Institut National du Cancer. The investigators obtained informed consent from each participant.

Open Access This article is distributed under the terms of the Creative Commons Attribution 4.0 International License (http://creativeco mmons.org/licenses/by/4.0/), which permits unrestricted use, distribution, and reproduction in any medium, provided you give appropriate credit to the original author(s) and the source, provide a link to the Creative Commons license, and indicate if changes were made.

\section{References}

1. Ivens D, Hoe AL, Podd TJ et al (1992) Assessment of morbidities from complete axillary dissection. Br J Cancer 66:136-138

2. Liljegren G, Holomberg L, The Uppsala Orebro Breast Study Group (1997) Arm morbidity after sector resection and axillary dissection with and without radiotherapy in breast cancer stage I. Results from a randomised trial. Eur J Cancer 33:193-199

3. Jackson JS, Olivotto IA, Wai EMDE et al (2000) A decision analysis of the effect of avoiding axillary lymph node dissection in low risk women with invasive breast carcinoma. Cancer 88:1852-1862

4. Giuliano AE, Kirgan DM, Guenther JM, Morton DL (1994) Lymphatic mapping and sentinel lymphadenectomy for breast cancer. Ann Surg 220:391-398

5. Kaplan EL, Meier P (1958) Nonparametric estimation from incomplete observations. J Am Stat Assoc 53:457-481

6. Cox DR (1972) Regression models and life-tables. J R Stat Soc Ser B Stat Methodol 34:187-220

7. Andersen PK, Klein JP, Zhang MJ (1999) Testing for centre effects in multi-centre survival studies: a Monte Carlo comparison of fixed and random effects tests. Stat Med 18:1489-1500

8. Veronesi U, Viale G, Paganelli G et al (2010) Sentinel lymph node biopsy in breast cancer: ten-year results of a randomized controlled study. Ann Surg 251:595-600 
9. Krag DN, Anderson SJ, Julian TB et al (2010) Sentinel-lymphnode resection compared with conventional axillary-lymph-node dissection in clinically node-negative patients with breast cancer: overall survival findings from the NSABP B-32 randomised phase 3 trial. Lancet Oncol 11:927-933

10. Mansel RE, Fallowfield L, Kissin M et al (2006) Randomized multicenter trial of sentinel node biopsy versus standard axillary treatment in operable breast cancer: the ALMANAC trial. J Natl Cancer Inst 98:599-609

11. Krag DN, Anderson SJ, Julian TB et al (2007) Technical outcomes of sentinel-lymph-node resection and conventional axillarylymph-node dissection in patients with clinically node-negative breast cancer: results from the NSABP B-32 randomised phase III trial. Lancet Oncol 8:881-888
12. Veronesi U, Paganelli G, Viale G et al (2003) A randomized comparison of sentinel-node biopsy with routine axillary dissection in breast cancer. N Engl J Med 349:546-553

13. Roy P, Estève J, Bobin JY (2000) Methodological questions in sentinel lymph node analysis in breast cancer patients. Ann Oncol 11:1381-1385

14. Kim T, Giuliano AE, Lyman GH (2006) Lymphatic mapping and sentinel node biopsy in early stage breast carcinoma. Cancer 106:4-16

15. Land SR, Kopec JA, Julian TB et al (2010) Patient-reported outcomes in sentinel node-negative adjuvant breast cancer patients receiving sentinel-node biopsy or axillar dissection: national surgical adjuvant breast and bowel project phase III protocol B-32. J Clin Oncol 28:3929-3936 